INTERVENTIONAL CARDIOLOGY AND SURGERY

\title{
Catheter ablation of permanent atrial fibrillation: medium term results
}

\author{
M J Earley, D J R Abrams, A D Staniforth, S C Sporton, R J Schilling

See end of article for authors' affiliations

.....................

Correspondence to: Dr Richard Schilling,

Cardiology Research Department, St Bartholomew's Hospital, Dominion House, 60 Bartholomew Close, London ECIA 7BE, UK r.schilling@qmul.ac.uk

Accepted 22 July 2005 Published Online First 23 August 2005

\begin{abstract}
Objective: To investigate the feasibility of catheter ablation as a treatment for symptomatic patients with longstanding permanent atrial fibrillation (AF).

Methods: Radiofrequency ablation was applied to encircle all pulmonary veins (PVs) and create lines from the left inferior PV to the mitral valve, along the roof of the left atrium between the PVs, and along the tricuspid valve-inferior vena cava isthmus. A seven day Holter was recorded at discharge and at follow up to assess arrhythmia burden. If patients developed a symptomatic, sustained atrial arrhythmia a repeat ablation procedure was advised.

Results: 42 patients underwent the procedure that took a mean of five hours with 50 minutes of fluoroscopy. After a median follow up of 8.4 months, 31 of 41 surviving patients $(76 \%)$ were in sinus rhythm. Of these, 29 patients were no longer taking any antiarrhythmic drugs but $22(52 \%)$ required more than one procedure. During follow up $49 \%$ experienced a sustained atrial tachycardia. Twenty six repeat procedures were performed. Maintenance of sinus rhythm after the first, second, or third procedure was $36 \%$ (15 of 42 ), $58 \%$ ( 11 of 19), and $71 \%$ (5 of 7), respectively. From a total of 68 procedures there were two serious complications (2.9\%): a stroke from which a full recovery was made, and a PV stenosis.

Conclusion: Catheter ablation can be used to cure longstanding permanent AF; however, there is a significant complication rate. Whether this is offset by a mortality benefit associated with sinus rhythm is unknown. Many patients will need more than one procedure to achieve success.
\end{abstract}

\section{METHODS \\ Inclusion criteria}

Curative AF ablation began at our institution in 2002 and the results of all consecutive patients with permanent AF treated between 5 April 2002 and 31 January 2005 are presented. To be included in the ablation programme patients had to be symptomatic, to have had unsuccessful treatment with at least one class 1 or 3 Vaughan-Williams antiarrhythmic drug, and to have undergone at least one direct current cardioversion. Permanent AF was defined according to the North American Society of Pacing and Electrophysiology/European Society of Cardiology international consensus as AF that had been present for some time and failure of cardioversion to restore sinus rhythm or reversion to AF within 24 hours. ${ }^{16}$ Patients had to have been in AF for three months continually to be included.

\section{Pre-ablation management}

All patients gave written informed consent for the procedure and had been taking warfarin for at least six weeks. The last dose was taken six days before the procedure and enoxaparin ( $1 \mathrm{mg} / \mathrm{kg}$ twice daily) was self administered subcutaneously for four days immediately before admission. ${ }^{17}$ A transoesophageal echocardiogram was recorded within 24 hours of the procedure to look for thrombus in the LA and appendage. After a transseptal puncture a bolus of intravenous unfractionated heparin (100 IU/kg) was given and an activated clotting time checked at 10 minutes and every 30 minutes subsequently. Further boluses were given to maintain an activated clotting time between 300 and 400 seconds. Femoral sheaths were removed after the procedure when the activated clotting time had dropped below 150 seconds.

\section{Ablation and electrophysiology procedure}

Two transseptal punctures were made to introduce two 8 French or an 8 French and 9 French sheath into the LA from the right femoral vein. All PVs were cannulated and baseline angiograms were recorded through the 8 French sheath. The

Abbreviations: AF, atrial fibrillation; LA, left atrium; PV, pulmonary vein 
mapping catheter was guided up each PV, which was tagged by a catheter location system. The catheter was moved around the LA under fluoroscopic guidance to allow reconstruction of the chamber (the "geometry"), labelling the PV os, mitral annulus, septum, and appendage. Care was taken to ensure accurate geometry collection between the ipsilateral PVs and between the appendage and left PVs. Three different systems were used to create the geometry and locate the catheters. In 25 patients non-contact (Ensite Array; Endocardial Solutions, St Paul, Minnesota, USA) and electroanatomical (Carto; Biosense Webster, Diamond Bar, California, USA) mapping were used in combination, in four Carto alone, and in the remaining 12 Ensite NavX (Endocardial Solutions).

Initially $4 \mathrm{~mm}$ and $8 \mathrm{~mm}$ radiofrequency ablation catheters were used (power $50 \mathrm{~W}, 60^{\circ} \mathrm{C}$ ). The power settings were altered after patient number 6 after we often observed char on the proximal end of the ablation electrode, particularly the $8 \mathrm{~mm}$ tip catheter, which culminated in the first significant complication. We then adopted catheters irrigated with heparin saline at $2 \mathrm{ml} / \mathrm{h}$ with the hope that this would prevent build up of char and reduce the risk of stroke, although there is no evidence to support this in the literature. Power, temperature, and irrigation flow rates were $20 \mathrm{~W}$, $50^{\circ} \mathrm{C}$, and $2 \mathrm{ml} / \mathrm{min}$ at the os of the PV; $30 \mathrm{~W}, 50^{\circ} \mathrm{C}$, and $2 \mathrm{ml} /$ min in the body of the LA; and $50 \mathrm{~W}, 45^{\circ} \mathrm{C}$, and $30 \mathrm{ml} / \mathrm{min}$ at the cavotricuspid isthmus. We did not use high flow irrigation during energy delivery because of data showing that lesion depth $(5.4 \mathrm{~mm})$ exceeds by some margin the thickness of the LA wall particularly at the venoatrial junction $(0.5-1 \mathrm{~mm}) .^{18} 19$

The ablation technique used evolved during the study. In the first 22 patients radiofrequency ablation was applied to encircle all PVs and create lines from the left inferior PV to the mitral valve and along the roof of the LA between the PVs (fig 1). At the end of the procedure a line of ablation was applied at the cavotricuspid isthmus in the right atrium during coronary sinus pacing until bidirectional conduction was blocked. Linear lesion integrity was assessed in all patients by the presence of widely split double potentials, coronary sinus catheter activation, and non-contact mapping when this was used. ${ }^{9}$ For the following 10 patients, in addition to the above procedure, a 14 pole adjustable PV mapping catheter (Orbiter PV; Bard EP, Lowell, Massachusetts, USA) was used to assess whether each PV was electrically isolated and to guide segmental ablation to achieve this where necessary. In the most recent 10 patients we performed all of the above, as well as targeting fractionated potentials mapped in the LA during AF.

\section{Follow up}

Patients were reloaded with warfarin and self administered enoxaparin until their international normalised ratio was therapeutic. Patients were discharged the day after the procedure with no specific antiarrhythmic medication prescribed. Patients' conditions were reviewed at six weeks and six months when a transthoracic and a transoesophageal (if in sinus rhythm) echocardiogram were recorded. Warfarin was stopped at six months if both LA mechanical function was restored and sinus rhythm was maintained, even though most of these patients were classed as at low risk of embolic stroke by international guidelines. ${ }^{20}$ At discharge and each follow up a seven day Holter recorder (Lifecard CF; Delmar Reynolds, Hertford, UK) was used to assess arrhythmia burden.

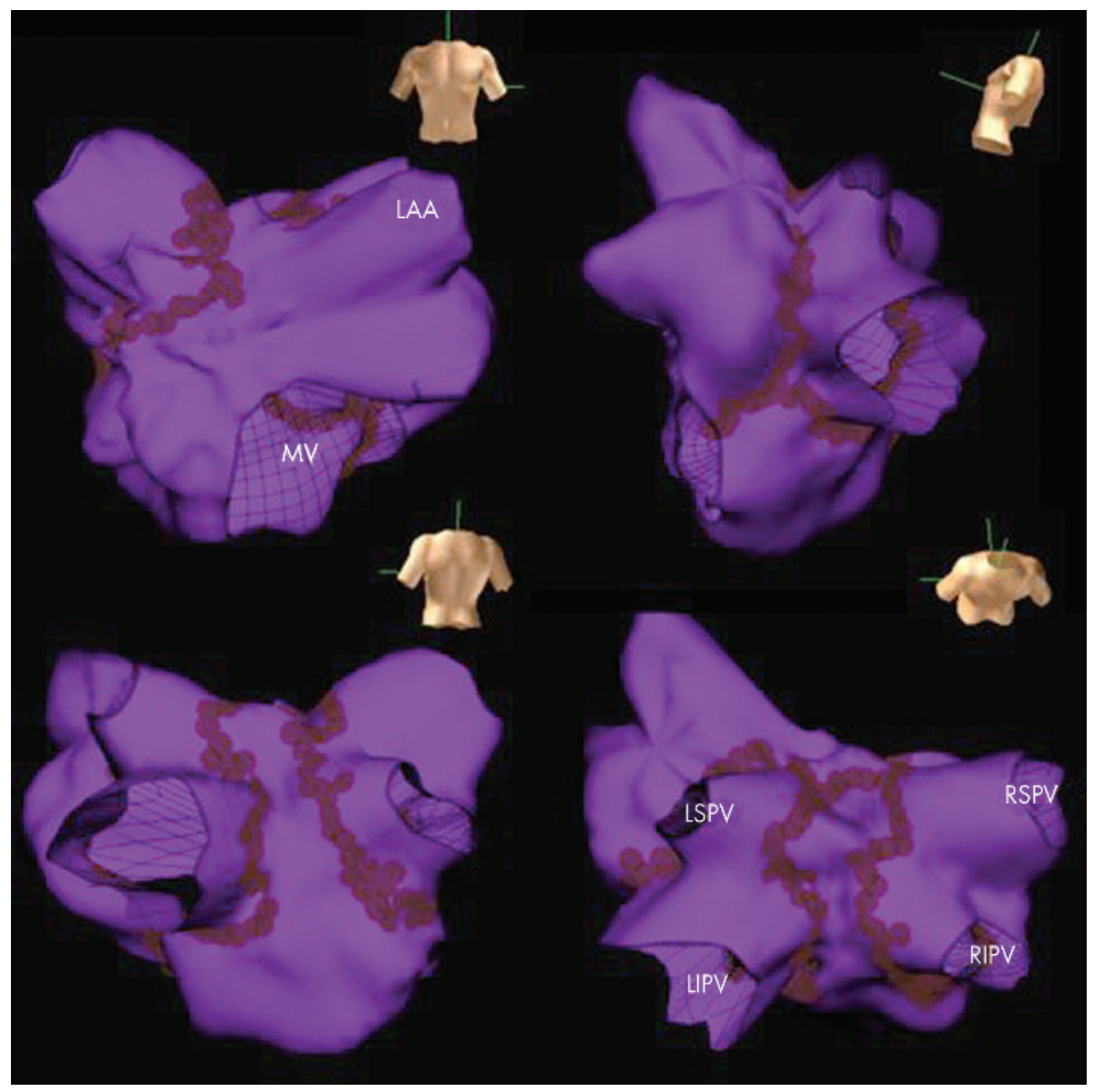

Figure 1 Endocardial surface (purple) of the left atrium (LA) recreated by the non-contact mapping system in one of the first 22 patients to undergo catheter ablation. The torso indicates the orientation of each view. Each brown mark represents a radiofrequency lesion. The standard set of lesions is applied to encircle the ipsilateral veins in pairs, to create lines between the left (LSPV) and right superior pulmonary veins (RSPV) across the roof and from the left inferior pulmonary vein (LIPV) to the mitral valve (MV) annulus. LAA, left atrial appendage; LSPV, left superior pulmonary vein. 


\begin{tabular}{ll} 
Table 1 Baseline clinical characteristics of the patients \\
\hline General characteristics & \\
Age (years) & $51(9)$ \\
Men/women & $36 / 6$ \\
Body mass index $\left(\mathrm{kg} / \mathrm{m}^{2}\right)$ & $31(5)$ \\
LA diameter $(\mathrm{mm})$ & $46(7)$ \\
AF history & \\
Antiarrhythmic drugs & $3.1(1.3)$ \\
DC cardioversions & $2.1(1.6)$ \\
Time since first AF episode (years) & $4.4(5)$ \\
Time since last in SR (months) & $13.6(18.9)$ \\
Cardiac pathology & 21 \\
None (lone AF) & 15 \\
Hypertension & 2 \\
Cardiomyopathy & 2 \\
Congenital heart disease & 2 \\
Aortic or mitral valve disease &
\end{tabular}

Data are presented as mean (SD) or number.

$A F$, atrial fibrillation; $D C$, direct current; LA, left atrial; SR, sinus rhythm.

Sustained arrhythmia was defined as atrial tachycardia or a fibrillation episode lasting greater than 24 hours on the Holter recording or captured on a 12 lead ECG at any time. If patients developed symptomatic, sustained atrial arrhythmia that persisted after the first follow up at six weeks, a repeat ablation procedure was advised. If the arrhythmia was atrial tachycardia the mechanism was elucidated by using a combination of electroanatomical (Carto) mapping and entrainment.

\section{Statistical analysis}

Unless stated otherwise, continuous and normally distributed data are expressed as mean (SD). Categorical data were compared by $\chi^{2}$ test and continuous data by Student's $t$ test adjusted for groups with unequal variances.

\section{RESULTS}

\section{Patients}

Table 1 describes the clinical characteristics of the 42 patients who underwent the procedure. Nine patients had previously undergone ablation: five cavotricuspid isthmus ablation for typical atrial flutter, three PV isolation for paroxysmal AF, and one a combination of these.

\section{Follow up}

Measured to the most recent appointment or hospital admission, the median (range) follow up since the first procedure was 8.4 (2-29) months.

\section{Overall success}

One patient died during follow up of an unrelated and previously undiagnosed tumour. Therefore, of the 41 surviving patients $31(76 \%)$ were in sinus rhythm with symptomatic improvement and no sustained arrhythmia on Holter monitoring, although three had non-sustained asymptomatic episodes of AF. Fourteen of these recovered LA function and maintained sinus rhythm for long enough to stop taking warfarin. Only two of these patients were taking any specific antiarrhythmic medication (both flecainide). Of the 10 patients not in sinus rhythm, three accepted permanent AF and seven ( six atrial tachycardia, one AF) are awaiting a further procedure. This is despite five repeat procedures performed in this group.

\section{Feasibility and safety of procedure}

In total, 68 procedures (including 26 repeat) were performed. Procedure length, screening time, and fluoroscopy dose area product were 314 (113) minutes, 50 (24) minutes, and 87 (70) $\mathrm{Gy} \cdot \mathrm{cm}^{2}$, respectively. There were two major $(2.9 \%)$ and three minor $(4.4 \%)$ complications. One patient had a stroke during the procedure manifest by left sided weakness, dysarthria, and dysphasia, which greatly improved after 30 minutes. At six months the symptoms had completely resolved. This complication precipitated a change to irrigated ablation catheters. Another patient had a symptomatic stenosis of the left superior PV that required angioplasty. The three minor complications were severe mechanical lumbar back pain, severe hypertension causing a large femoral haematoma, and a pericardial effusion that resolved spontaneously.

\section{Arrhythmia recurrences}

To achieve sinus rhythm 13 of 31 (42\%) patients required one and four of 31 (13\%) required two repeat ablation procedures. Ten $(24 \%)$ patients remained free of sustained arrhythmia after their index procedure with most recurrences occurring in the first few weeks. In addition to sustained arrhythmia seven day Holter recordings showed that three patients had asymptomatic non-sustained episodes of AF. The sustained recurrences were atrial tachycardia in eight (20\%), AF in $12(29 \%)$, and a combination in $12(29 \%)$. Patients with recurrence were older and had longer duration of AF. Neither LA size nor the presence of structural heart disease predicted early recurrence of AF (table 2). A procedure that included the use of a PV catheter to confirm isolation of the PV or the targeting of fragmented potentials during AF did not predict freedom from sustained recurrence (table 2). ${ }^{21}$

\begin{tabular}{|c|c|c|c|}
\hline Predictor & $\begin{array}{l}\text { No recurrence } \\
n=16(38 \%)\end{array}$ & $\begin{array}{l}\text { Recurrence } \\
n=26(62 \%)\end{array}$ & $\mathrm{p}$ Value \\
\hline \multicolumn{4}{|l|}{ General } \\
\hline Age (years) & $48(11)$ & $54(7)$ & 0.04 \\
\hline Men/women & $15 / 2$ & $21 / 1$ & 0.70 \\
\hline \multicolumn{4}{|l|}{ AF history } \\
\hline Antiarrhythmic drugs & $3.0(1.5)$ & $3.1(1.1)$ & 0.9 \\
\hline Time since first AF episode (months) & $31(20)$ & $65(72)$ & $<0.01$ \\
\hline \multicolumn{4}{|l|}{ Cardiac pathology } \\
\hline Structural heart disease & 7 & 14 & 0.32 \\
\hline LA diameter $(\mathrm{mm})$ & $46(9)$ & $46(6)$ & 0.30 \\
\hline \multicolumn{4}{|l|}{ Ablation technique } \\
\hline Addition of segmental PV isolation & $9(45 \%)$ & $11(55 \%)$ & 0.38 \\
\hline Targeting of fragmented signals & $5(50 \%)$ & $5(50 \%)$ & 0.37 \\
\hline
\end{tabular}




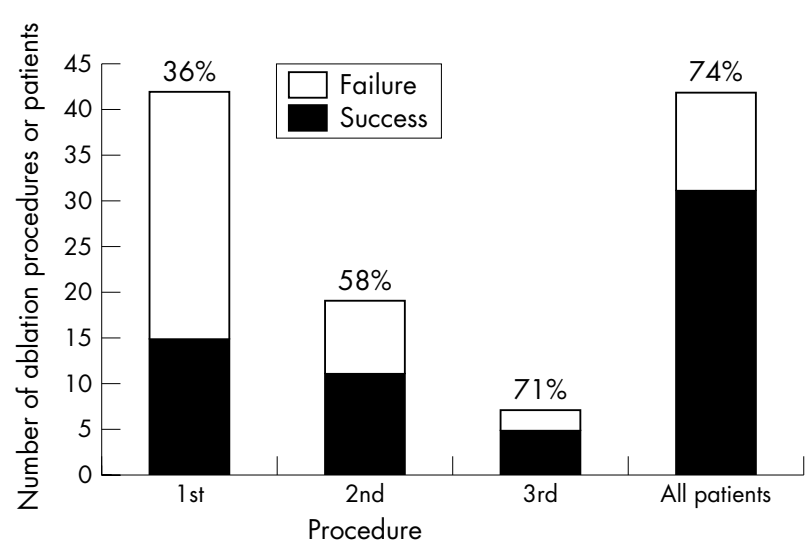

Figure 2 Graphical representation of maintenance of sinus rhythm after radiofrequency ablation procedures. Of all 42 patients (right hand column) $74 \%$ maintained sinus rhythm, although half required more than one procedure. The likelihood of maintaining sinus rhythm without the need for a repeat ablation after the first, second, and third procedure is $36 \%$ (15 of 42), $58 \%$ (11 of 19), and $71 \%$ (5 of 7), respectively.

\section{Repeat procedures}

A total of 20 second and six third procedures have been performed. These 26 procedures consisted of 12 repeat catheter maze procedures (with additional right atrial lines, PV isolation, and fractionated potential ablation), 11 atrial tachycardia ablations, and three segmental PV isolations. Repeat procedures were not significantly shorter than the index procedure $(279 v 335$ minutes, $\mathrm{p}=0.06)$. The probability of maintaining sinus rhythm without the need for a further ablation after the first, second, and third procedure was $36 \%$ ( 15 of 42 ), $58 \%$ ( 11 of 19 ), and $71 \%$ (five of seven), respectively (fig 2 ).

In 23 of the 26 repeat procedures the LA was mapped. In 15 (65\%), none of the four veins were shown to be isolated and in only one patient $(4 \%)$ were all veins isolated. Surprisingly the use of a PV catheter did not significantly increase the rate of PV isolation at the repeat procedure.

\section{Atrial tachycardias}

In all patients with atrial tachycardia the right atrium was mapped initially to avoid unnecessary transseptal puncture. Three tachycardias were identified as macro-reentrant right atrial tachycardia with conduction through the tricuspid valve-inferior vena cava isthmus (typical atrial flutter). In the remaining eight patients, two tachycardias were focal atrial tachycardia emerging from the left superior PV, four were macro-reentrant, and in two the mechanism could not be determined. The re-entry circuits identified were a clockwise circuit around the mitral valve in three and in one a circuit around a small area of scar on the medial aspect of the anterior LA wall. In all cases where a definite mechanism was identified the tachycardia terminated during ablation.

\section{DISCUSSION}

\section{Main findings}

This study investigated the feasibility of percutaneous catheter ablation incorporating LA linear lesions and PV isolation for the treatment of patients with longstanding permanent AF. We have shown that most (76\%) patients can have sinus rhythm restored and maintained by medium term follow up. The rate of recurrence of both $\mathrm{AF}$ and atrial tachycardia is high. However, these can be successfully managed with a repeat ablation procedure (fig 2). Longer duration of AF predicts early recurrence of sustained arrhythmia and therefore affected patients are even more likely to need multiple procedures to achieve maintenance of sinus rhythm.

\section{Safety}

We attributed the stroke to the accumulation of char on the tip of the ablation catheter. Ernst et $a^{14}$ reported an incidence of $2.4 \%$ for thromboembolic complications in 84 patients undergoing extensive LA ablation with a $4 \mathrm{~mm}$ non-irrigated catheter. Macle et $a l^{5}$ used an irrigated catheter in $136 \mathrm{PV}$ segmental isolation cases without any thromboembolic episodes. Since adopting this technique we have had no other strokes in any subsequent AF ablation performed at our institution. The PV stenosis occurred in a patient who had previously undergone focal ablation procedures targeting the PV, complicated by a mild stenosis. Even though we were using electroanatomical mapping and PV angiography to identify the catheter position to ablate outside the ostium, we hypothesise that the catheter must have slipped into the vein during energy delivery resulting in stenosis.

\section{Catheter ablation for permanent AF}

The efficacy and safety of ablation for paroxysmal AF with an end point of either electrical isolation or voltage reduction within the PV has been established. ${ }^{62}$ Only two studies have looked exclusively at this strategy for permanent AF and reported a one year success of $21 \%$ and $60 \%$ of patients ceasing antiarrhythmic drugs. ${ }^{73}$ However, other studies have reported a mixture of paroxysmal and permanent AF with the approach of circumferential lesions outside the vein. Pappone et $a l^{6}$ reported 72 patients with permanent AF achieving 68\% freedom from AF compared with 85\% for paroxysmal AF. Success was related to the volume of LA tissue enclosed in these lines, suggesting that reduction (debulking) of myocardium available to fibrillate is as important as isolation of the veins. Other studies that examined non-paroxysmal AF (which included permanent and persistent AF) have shown similar results. ${ }^{15}$ The results of circumferential PV isolation alone in permanent AF have been disappointing, raising the possibility that wider encirclement or the addition of linear lesions is important to abolish permanent AF with results similar to those with the surgical maze..$^{7124}$

Although linear lesions limited to the right atrium are safer, they have a low effectiveness in preventing recurrences in permanent $\mathrm{AF} .{ }^{25}$ Delivering long linear lesions in the LA and ensuring completeness of these lines is technically difficult, needing mean procedure times of eight hours, although this technique improves outcomes. ${ }^{914}$ Pappone et $a l^{27}$ reported, however, that in 280 patients $(39 \%$ with permanent $\mathrm{AF}$ ) a technique of circumferential ablation outside the vein combined with two lines along the posterior LA between the PVs and linking the left PVs to the mitral valve can be performed in 110 minutes, showing the feasibility of such an approach.

Ablation around the PVs remains the key element of AF ablation, as it isolates $\mathrm{PV}$ extrasystoles that may initiate $\mathrm{AF}^{28}$ reduces the potential of local re-entry circuits at the PV ostia, which may act as mother rotors, ${ }^{29}$ and modulates the local autonomic nerve system by destroying vagal nerve endings epicardially. ${ }^{30}$ However, long term success is improved with the addition of linear LA lesions, particularly in permanent $\mathrm{AF}$, and explains why high success rates such as we have presented can be achieved. ${ }^{27}$

\section{Recurrences}

We report a very high incidence of recurrence of sustained arrhythmia $(76 \%)$ after the initial procedure of which the majority occurred within the first eight weeks. Forty nine per cent of patients experienced a sustained atrial tachycardia. 
Comparing these results with other published data is difficult for several reasons. The incidence of atrial tachycardia after ablation for paroxysmal AF is reported to be between 3-10\%; however, in all these studies recurrences occurring in the first 6-8 weeks are ignored unless they persist beyond this period. ${ }^{27} 3132$ No previous study has published results of linear ablation exclusively in permanent AF, but small studies have shown the importance of demonstrable conduction block. ${ }^{914}$ Lastly, large observational studies usually look at a heterogeneous group of patients and, although they recognise permanent $\mathrm{AF}$ as a predictor of recurrence, it is not always possible to separate the results. For example, Pappone et $a^{27}$ showed that in 280 patients (39\% with permanent AF) the rates of atrial tachycardia $(3.9 \%)$ and AF (12.9\%) were very low; however, over $90 \%$ of the recurrences occurred in patients with permanent AF.

Incomplete lines act as a substrate for re-entry and are associated with atrial tachycardia recurrence. ${ }^{914}$ Gaps in ablation lines as small as $2 \mathrm{~mm}$ can permit normal conduction and therefore a strategy of delivering long linear lesions in the left atria, by using lower powers than in previous studies, may explain this high rate of atrial tachycardia. $^{33}$ The alternative mechanism is recovery of conduction between the LA and PVs, which has been identified as a reason for recurrences in paroxysmal AF. ${ }^{3134}$

\section{Limitations}

Our procedure times were still very long, reflecting a learning curve for performing these complex procedures and in following a research protocol. We did not use a consistent protocol throughout this study, as our procedure evolved to include effective techniques such as targeting complex fractionated potentials. ${ }^{21}$ Our results have shown an improvement on previously published data for permanent AF.

\section{Conclusions}

We have shown that catheter ablation can be used to cure AF but there are several important limitations to this procedure. Firstly, the rate of complications, including stroke, is significant. Whether this is offset by a mortality benefit associated with sinus rhythm remains to be determined in randomised controlled trials. Secondly, many patients will need more than one procedure to achieve a $75 \%$ success rate; whether this improves with developments in technique and technology remains to be seen.

\section{ACKNOWLEDGEMENTS}

Dr Earley is the recipient of a British Heart Foundation Junior Research Fellowship (FS/02/078/14748).

\section{Authors' affiliations \\ M J Earley, D J R Abrams, A D Staniforth, S C Sporton, R J Schilling, St Bartholomew's Hospital, London, UK \\ Conflict of interest: Dr Earley has received payment for a lecture given on behalf of Endocardial Solutions. Dr Schilling is a member of the scientific advisory board for Biosense Webster. He is listed on the speakers' bureau for Endocardial Solutions and has received payment for lectures sponsored by them. He has received sponsorship for travel to international meetings from Guidant, Medtronic, St Jude Medical, Endocardial Solutions, and Biosense Webster. The other authors have no specific competing interests other than support for travel to international meetings from Guidant, Medtronic, St Jude Medical, Endocardial Solutions, and Biosense Webster.}

\section{REFERENCES}

1 Benjamin EJ, Wolf PA, D'Agostino RB, et al. Impact of atrial fibrillation on the risk of death: the Framingham heart study. Circulation 1998;98:946-52.

2 Carlsson J, Miketic S, Windeler J, et al. Randomized trial of rate-control versus rhythm-control in persistent atrial fibrillation: the strategies of treatment of atrial fibrillation (STAF) study. J Am Coll Cardiol 2003;41:1690-6.
3 The AFFIRM Investigators. Relationships between sinus rhythm, treatment, and survival in the atrial fibrillation follow-up investigation of rhythm management (AFFIRM) study. Circulation 2004;109:1509-13.

4 Cappato R, Calkins H, Chen SA, et al. Worldwide survey on the methods, efficacy, and safety of catheter ablation for human atrial fibrillation. Circulation 2005; 111:1100-5.

5 Macle L, Jais P, Weerasooriya R, et al. Irrigated-tip catheter ablation of pulmonary veins for treatment of atrial fibrillation. $J$ Cardiovasc Electrophysiol 2002;13:1067-73.

6 Pappone C, Oreto G, Rosanio S, et al. Atrial electroanatomic remodeling after circumferential radiofrequency pulmonary vein ablation: efficacy of an anatomic approach in a large cohort of patients with atrial fibrillation. Circulation 2001; 104:2539-44.

7 Haissaguerre M, Jais P, Shah DC, et al. Catheter ablation of chronic atrial fibrillation targeting the reinitiating triggers. J Cardiovasc Electrophysiol 2000;11:2-10.

8 Natale A, Pisano E, Beheiry S, et al. Ablation of right and left atrial premature beats following cardioversion in patients with chronic atrial fibrillation refractory to antiarrhythmic drugs. Am J Cardiol 2000;85:1372-5.

9 Seidl K, Schwacke H, Zahn R, et al. Catheter ablation of chronic atrial fibrillation with noncontact mapping: are continuous linear lesions associated with ablation success? Pacing Clin Electrophysiol 2003;26:534-43

10 Nattel S. New ideas about atrial fibrillation 50 years on. Nature 2002;415:219-26.

11 Cox JL, Ad N, Palazzo T, et al. Current status of the maze procedure for the treatment of atrial fibrillation. Semin Thorac Cardiovasc Surg 2000;12:15-9.

12 Swartz JF, Pellersels G, Silvers J, et al. A catheter based curative approach to atrial fibrillation in humans [abstract]. Circulation 1994;90:1335.

13 Cannom DS. Atrial fibrillation: nonpharmacologic approaches. Am J Cardiol 2000;85:25D-35D.

14 Ernst S, Ouyang F, Lober F, et al. Catheter-induced linear lesions in the left atrium in patients with atrial fibrillation: an electroanatomic study. J Am Coll Cardiol 2003:42:1271-82.

15 Verma A, Wazni OM, Marrouche NF, et al. Pre-existent left atrial scarring in patients undergoing pulmonary vein antrum isolation: an independent predictor of procedural failure. J Am Coll Cardiol 2005:45:285-92.

16 Levy S, Camm AJ, Saksena S, et al. International consensus on nomenclature and classification of atrial fibrillation: aA collaborative project of the Working Group on Arrhythmias and the Working Group of Cardiac Pacing of the European Society of Cardiology and the North American Society of Pacing and Electrophysiology. J Cardiovasc Electrophysiol 2003; 14:443-5.

17 Stellbrink C, Nixdorff U, Hofmann T, et al. Safety and efficacy of enoxaparin compared with unfractionated heparin and oral anticoagulants for prevention of thromboembolic complications in cardioversion of nonvalvular atrial fibrillation: the anticoagulation in cardioversion using enoxaparin (ACE) trial. Circulation 2004; 109:997-1003.

18 Ho SY, Cabrera JA, Tran VH, et al. Architecture of the pulmonary veins: relevance to radiofrequency ablation. Heart 2001;86:265-70.

19 Nakagawa H, Wittkampf FH, Yamanashi WS, et al. Inverse relationship between electrode size and lesion size during radiofrequency ablation with active electrode cooling. Circulation 1998;98:458-65.

20 Fuster V, Ryden LE, Asinger RW, et al. ACC/AHA/ESC guidelines for the management of patients with atrial fibrillation: executive summary a report of the American College of Cardiology/American Heart Association task force on practice guidelines and the European Society of Cardiology committee for practice guidelines and policy conferences (committee to develop guidelines for the management of patients with atrial fibrillation) developed in collaboration with the North American Society of Pacing and Electrophysiology. Circulation 2001;104:2118-50.

21 Nademanee K, McKenzie J, Kosar E, et al. A new approach for catheter ablation of atrial fibrillation: mapping of the electrophysiologic substrate. J Am Coll Cardiol 2004;43:2044-53.

22 Haissaguerre $M$, Jais $P$, Shah DC, et al. Electrophysiological end point for catheter ablation of atrial fibrillation initiated from multiple pulmonary venous foci. Circulation 2000;101:1409-17.

23 Kanagaratnam L, Tomassoni G, Schweikert R, et al. Empirical pulmonary vein isolation in patients with chronic atrial fibrillation using a three-dimensional nonfluoroscopic mapping system: long-term follow-up. Pacing Clin Electrophysiol 2001;24:1774-9.

24 Gaita F, Riccardi R, Caponi D, et al. Linear cryoablation of the left atrium versus pulmonary vein cryoisolation in patients with permanent atrial fibrillation and valvular heart disease: correlation of electroanatomic mapping and long-term clinical results. Circulation 2005;111:136-42.

25 Ernst S, Schluter M, Ouyang F, et al. Modification of the substrate for maintenance of idiopathic human atrial fibrillation: efficacy of radiofrequency ablation using nonfluoroscopic catheter guidance. Circulation 1999: 100:2085-92.

26 Calo L, Lamberti F, Loricchio ML, et al. Long-term follow-up of right atrial ablation in patients with atrial fibrillation. J Cardiovasc Electrophysiol 2004; 15:37-43.

27 Pappone C, Manguso F, Vicedomini G, et al. Prevention of iatrogenic atrial tachycardia after ablation of atrial fibrillation. a prospective randomized study comparing circumferential pulmonary vein ablation with a modified approach. Circulation 2004;1 10:3036-42. 
28 Haissaguerre $M$, Jais $P$, Shah DC, et al. Spontaneous initiation of atrial fibrillation by ectopic beats originating in the pulmonary veins. N Engl J Med 1998;339:659-66.

29 Jalife J, Berenfeld O, Mansour M. Mother rotors and fibrillatory conduction: a mechanism of atrial fibrillation. Cardiovasc Res 2002;54:204-16.

30 Pappone C, Santinelli V, Manguso F, et al. Pulmonary vein denervation enhances long-term benefit after circumferential ablation for paroxysmal atrial fibrillation. Circulation 2004; 109:327-34.

31 Gerstenfeld EP, Callans DJ, Dixit S, et al. Mechanisms of organized left atrial tachycardias occurring after pulmonary vein isolation. Circulation 2004; 110:1351-7.
32 Kilicaslan F, Verma A, Yamaji H, et al. The need for atrial flutter ablation following pulmonary vein antrum isolation in patients with and without previous cardiac surgery. J Am Coll Cardiol 2005;45:690-6.

33 Mitchell MA, McRury ID, Evereft TH, et al. Morphological and physiological characteristics of discontinuous linear atrial ablations during atrial pacing and atrial fibrillation. J Cardiovasc Electrophysiol 1999;10:378-86

34 Ouyang $F$, Antz $M$, Ernst $S$, et al. Recovered pulmonary vein conduction as a dominant factor for recurrent atrial tachyarrhythmias after complete circular isolation of the pulmonary veins: lessons from double lasso technique. Circulation 2005;111:127-35.

\section{IMAGES IN CARDIOLOGY}

\section{Left atrial obliteration after coronary artery perforation}

doi: $10.1136 /$ hrt.2005.066621

A 78 year old man with inducible ischaemia on myocardial perfusion imaging and without prior cardiac disease underwent percutaneous coronary intervention of a severe stenosis of the left circumflex artery. Attempts to cross the lesion with the guidewire failed and the vessel occluded. After recanalisation with a different guidewire, a balloon could not be advanced across the lesion and the procedure was aborted. At this time, there was faint myocardial blushing adjacent to the lesion (panel A). One hour later atrial fibrillation with fast ventricular rate and arterial hypotension developed. Emergency transthoracic echocardiography did not show a pericardial effusion. Several attempts at electrical cardioversion after intravenous amiodarone failed. The patient was ventilated and received vasopressors for haemodyamic support. Sinus rhythm was finally restored by fluid administration and a continuous infusion of esmolol. Transoesophageal echocardiography (TOE) revealed a mass obliterating the left atrial cavity (panels $\mathrm{B}$ and $\mathrm{C}$, video $\mathrm{l}$; to view video footage visit the Heart websitehttp://www.heartjnl.com/supplemental).

Emergency surgical drainage evacuated a haemorrhagic pericardial effusion and the haemodynamic status improved.
A follow up TOE (panel D) and computed tomography showed residual haematoma of the lateral wall of the left atrium. Left and right heart diastolic pressures were equilibrated (panel E) and the cardiac index was $1.9 \mathrm{l} / \mathrm{min}$ / $\mathrm{m}^{2}$ - findings compatible with residual tamponade. Careful medical management with administration of dobutamine could avoid a second surgical intervention.

This case shows that a localised cardiac tamponade can be the cause of cardiogenic shock after documented coronary perforation, even in patients without prior cardiothoracic surgery, and that wall haematoma can cause tamponade physiology.
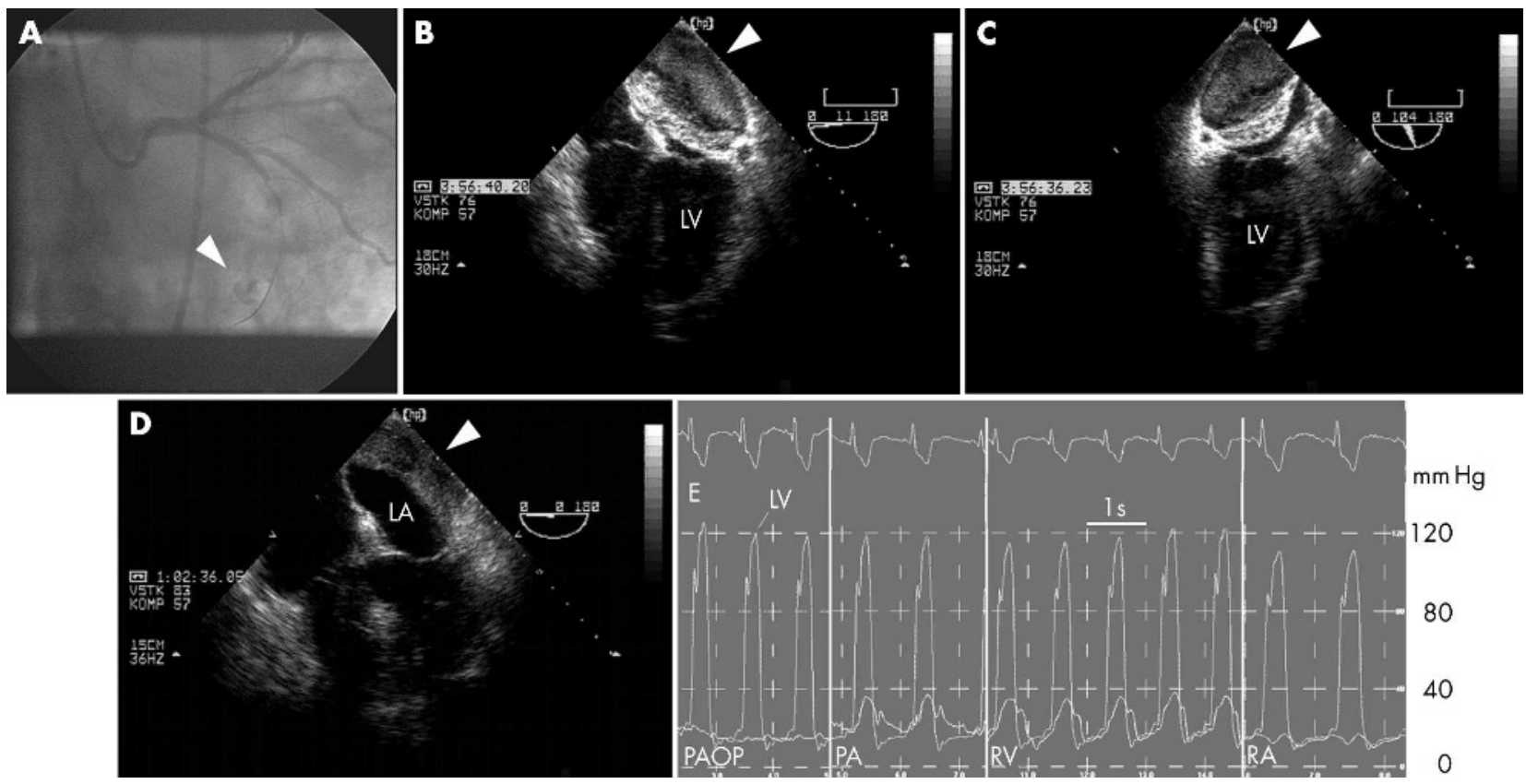